\section{Chromoblastomycosis evolving to sarcomatoid squamous cell carcinoma: A case report}

\author{
Walter Belda Junior, ${ }^{1,2}$ Paulo Ricardo \\ Criado, ${ }^{3}$ Paula Casteleti, ${ }^{1}$ Luiz Felipe \\ Domingues Passero ${ }^{4,5}$
}

${ }^{1}$ Dermatology Department, University of São Paulo, Medical School, Clinics Hospital, São Paulo; ${ }^{2}$ Laboratory of Pathology of Infectious Diseases, Medical School, University of São Paulo, São Paulo; ${ }^{3}$ ABC School of Medicine, Fundação Universitária do $\mathrm{ABC}$ (FUABC), Santo André; ${ }^{4}$ Institute of Biosciences, São Paulo State University (UNESP), São Vicente; ${ }^{5}$ Institute for Advanced Studies of Ocean, São Paulo State University (UNESP), São Vicente, Brazil

\begin{abstract}
Chromoblastomycosis (CMB) is a cutaneous fungal infection caused by dematiaceous fungi. According to the World Health Organization $\mathrm{CMB}$ has been elected as a tropical disease, and it is prevalent in tropical and subtropical regions. The lower extremities are the most affected areas, and the lesions progress with erythema, papules, nodules, verrucose plates and/or ulcerations. So far, few works have demonstrated neoplastic transformation in chronic $\mathrm{CMB}$ lesions, and it may be a consequence of prolonged inflammatory response. In the present case report, we described a neoplastic transformation from CMB lesion of a 55year-old man, presenting lesions only in the left leg for 35 years. After treatment, a verrucous white plate with thick and irregular borders emerged in the ankle, which was identified as a sarcomatoid squamous cell carcinoma. The present case report highlights the importance of an early diagnosis and treatment.
\end{abstract}

\section{Introduction}

Chromoblastomycosis (CBM) is a progressive and disabling cutaneous fungal infection, caused by dematiaceous fungi that belong to the order Chaetothyriales and family Herpotrichiellaceae; the most important etiologic agents are the saprophytic fungi Fonsecaea pedrosoi and Cladophialophora carrionnii. This infection affects mainly people living in tropical areas, additionally the efficacy of drugs and therapeutic options to treat $\mathrm{CBM}$ are limited. CBM has been studied since 40 's, ${ }^{1}$ however, little is known about the neoplastic transformation of this mycotic infection. ${ }^{2}$ In the present work, we reported the existence of a large sarcomatoid squamous carcinoma behind the CBM lesion in a patient, suggesting neoplastic transformation of the chronic skin lesion.

\section{Case Report}

A 55-year-old man, farmer, had lesions only in the left leg for 35 years. He was born in Atibaia city, São Paulo, Brazil, but the skin lesions appeared while working in the mining activity, Rondonia State, Brazil. The patient went to the medical services and was submitted to different therapeutic methods, such as itraconazole, fluconazole, ketoconazole, terbinafine, cryotherapy and phototherapy, but with limited success. Medical records detailing these previous treatments could not be found. He was admitted to the Dermatology outpatient service of the "Hospital das Clínicas da Universidade de São Paulo". At the time of our evaluation, an extensive verrucous lesion affecting almost the entire left leg was verified, however lesions were not observed in the right leg or anywhere else on the body. In the left leg, numerous skin lesions were detected, and they were erythematous, infiltrated, circumscribed with irregular plaques and on the skin surface, lesions were hyperkeratotic - verrucous type (Figure 1A and B). Verrucous plates and sclerotic bodies were observed in the dorsal area of the foot (Figure 1B). Muriform cells were identified in the skin histological sections stained by hematoxylin and eosin (Figure 1C). Skin smear culture, carried out in Sabouraud dextrose agar medium, led to the morphological identification of a Fonsecaea sp., as the etiological agent after the $15^{\text {th }}$ day of culture. Levels of blood AST, ALT and creatinine levels were normal, and the treatment with the drug combination was initiated with oral itraconazole $(200 \mathrm{mg} / \mathrm{kg})$ and acitretin $(50 \mathrm{mg} / \mathrm{kg})$, once a day; along with topical imiquimod (Modik ${ }^{\circledR}-50 \mathrm{mg} / \mathrm{g}$ ) once a day, five times a week. ${ }^{3}$ Previously it was demonstrated that acitretin associated to the classic therapy was effective at controlling CMB lesions, ${ }^{4}$ thus this combination was elected to treat such patient, even because monotherapy or physical approaches were not efficient. After four months of treatment significant reduction of hyperkeratotic and verrucous plates were observed (Figure 1D), and simultaneously a verrucous white
Correspondence: Walter Belda Jr., Dermatology Department, University of São Paulo, Medical School, Clinics Hospital, São Paulo, Brazil

Tel.: +551130618339.

E-mail: walterbelda@uol.com.br

Key words: Chromoblastomycosis; carcinoma; treatment.

Acknowledgments: The authors would like to thank HCFMUSP-LIM50.

Contributions: conceptualization: WBJ; PRC; formal analysis: WBJ; PRC; PC; LFDP; investigation: WBJ; PRC; PC; resources: WBJ; PRC; LFDP; data curation: WBJ; writing: WBJ; PRC; LFDP; project administration: WBJ.

Conflict of interest: The authors declare no potential conflict of interest.

Funding: None.

Ethics approval: Approved

Consent to publication: Received.

Availability of data and materials: Available upon request

Please cite this article as: Belda W jr, Criado $P R$, Casteleti P, Domingues Passero LF. Chromoblastomycosis evolving to sarcomatoid squamous cell carcinoma: A case report. Dermatol Rep 2021;13:9009.

Received for publication: 6 November 2020 Revision received: 15 December 2020.

Accepted for publication: 16 December 2020.

This work is licensed under a Creative Commons Attribution-NonCommercial 4.0 International License (CC BY-NC 4.0).

${ }^{\circ}$ Copyright: the Author(s), 2021

Licensee PAGEPress, Italy

Dermatology Reports 2021; 13:9009

doi:10.4081/dr.2021.9009

plate with thick and irregular borders in the ankle emerged (Figure 1E), suggesting the presence of a tumor under the skin lesions. The association of acitretin, itraconazole and imiquimod did not cause changes in the levels of AST, ALT and creatinine. Histopathological study of this area showed the presence of a squamous cell carcinoma (SC), as showed in the figures $2 \mathrm{~A}$ and $\mathrm{B}$. Histological sections of the skin immunolabeled with anti-CD68 (Figure 2C), anticytokeratin (34 $\square$ E12) (Figure 2D) and antivimentin (Figure 2E) antibodies showed positivity for all analyzed molecules. The antibodies anti-vimentin and anti-CD68 have been considered as important markers 
to identify carcinomas, ${ }^{5-7}$ reinforcing that this patient had a squamous cell carcinoma; additionally, anti-34 $\square$ E12 (cytokeratin), that is the most sensitive marker to identify sarcomatoid subsets of tumors, ${ }^{8}$ was detected in the skin biopsy of aforementioned patient, leading to the identification of sarcomatoid subsets in his skin.

Nuclear magnetic resonance displayed a diffuse thickness of the skin and subcutaneous tissue. A large neoplastic lesion was observed in the lateral and dorsal areas of the left feet, with bone infiltration (data not shown). Patient was subjected to 13 cycles of carboplatin and paclitaxel therapy, which were ineffective at controlling neoplastic lesions. Ultrasonographic analysis and fine needle aspiration puncture showed no lymph node involvement, and the left leg was amputated. The patient had a good clinical recovery and no tumor recurrence has been observed so far. Although combined therapy leads to a great improvement of the lesions in the superior area of the left leg (Figure 1D), CMB still persists, and this patient is still under monitoring.

\section{Discussion}

During the evolution of CMB frequent complications have been observed and are associated with secondary bacterial infections, appearance of chronic ulcerations, lymphedema and chronic nonhealing ulcers. Extracutaneous involvement of CBM has also been described and affects the bones, lymph nodes, brain and lungs. In persistent infection, malignant transformation has been observed and some authors have already reported the malignant transformation to squamous cell carcinoma. ${ }^{2} \mathrm{~A}$ delay in healthcare seeking of a few months or years may account to the pathological manifestations of a noninfectious nature, such as neoplastic transformation. The case presented here has 35 years of evolution, and possibly the chronification of the lesions as well as the inflammatory response, lead to the appearance of a squamous cell carcinoma. The first report of malignant transformation of CBM lesions, described by Caplan in $1968,{ }^{9}$ evolved over 11 years. Although the pathophysiological basis of the malignant transformation has not been fully elucidated, ${ }^{8}$ the presence of polymorphonuclear cells and activated macrophages may promote the release of enzymes and free radicals that may trigger malignant transformation over the years. Although malignant transformation of chronic CMB lesions has previously been demonstrated the rate of neoplastic transformation is low. ${ }^{910}$ In India, only 1 out 70 patients with chromoblastomycosis developed squamous cell carcinoma, ${ }^{11}$ similarly in Mexico from 51 patients analyzed, carcinoma was identified in only 1 patient. ${ }^{12}$

\section{Conclusions}

This case report highlights the importance of early diagnosis of CMB infection as well as efficient treatment, avoiding the chronification of lesions and tumor development, that in fact can bring more quality of life to the patient and also reduce the mortality induced by the skin cancer.
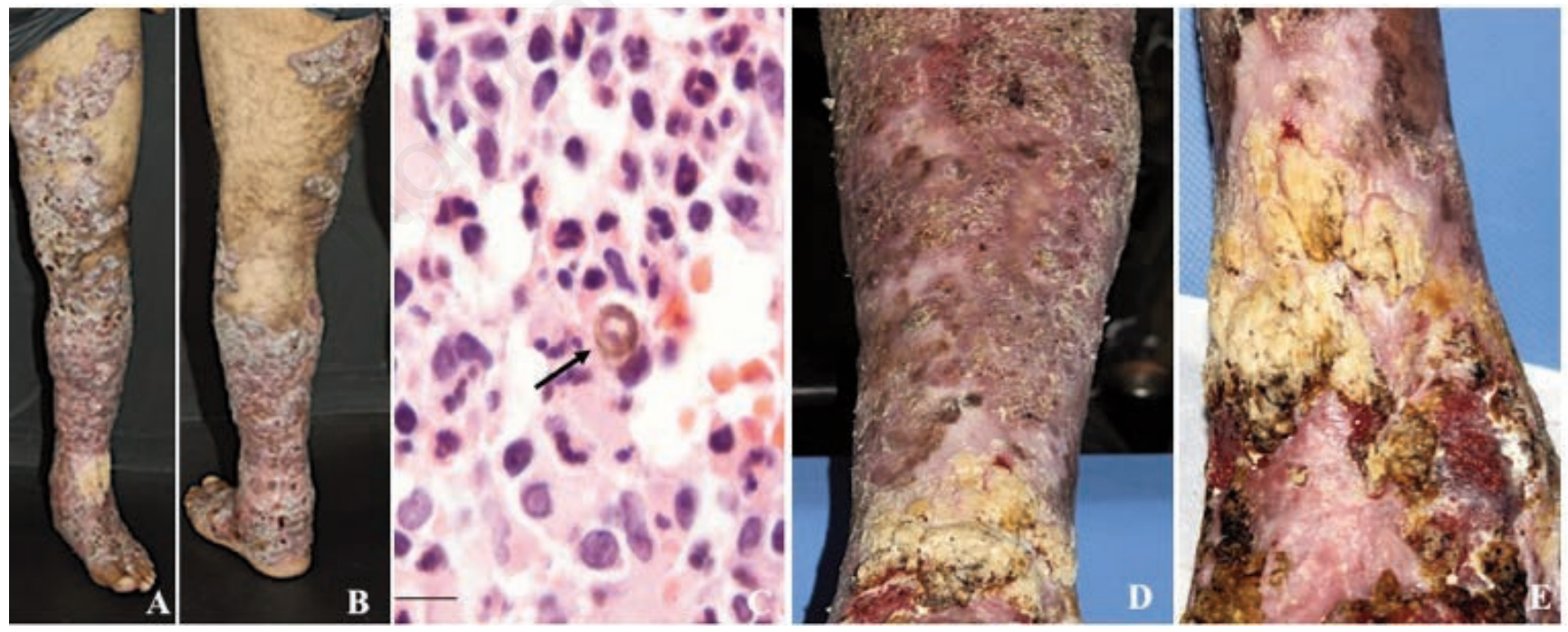

Figure 1. Patients with chromoblastomycosis presented numerous skin lesions in the frontal (A) and dorsal area of the left leg (B), characterized by irregular erythematous, infiltrated, circumscribed, plaques with hyperkeratosis in the inferior members. Histological section of the skin stained by hematoxylin and eosin allows the identification of muriform cells (Figure $\mathrm{C}$ ). Patient was treated with the combination of oral itraconazole $\left(200 \mathrm{mg} /\right.$ day) and acitretin $(50 \mathrm{mg} / \mathrm{kg})$, as well as topical imiquimod (Modik $\left.{ }^{\circledR}-50 \mathrm{mg} / \mathrm{g}\right)$ and a significant reduction of the skin lesions were observed (D). After four months of combined treatment a verrucous white plate in the ankle emerged (E). 


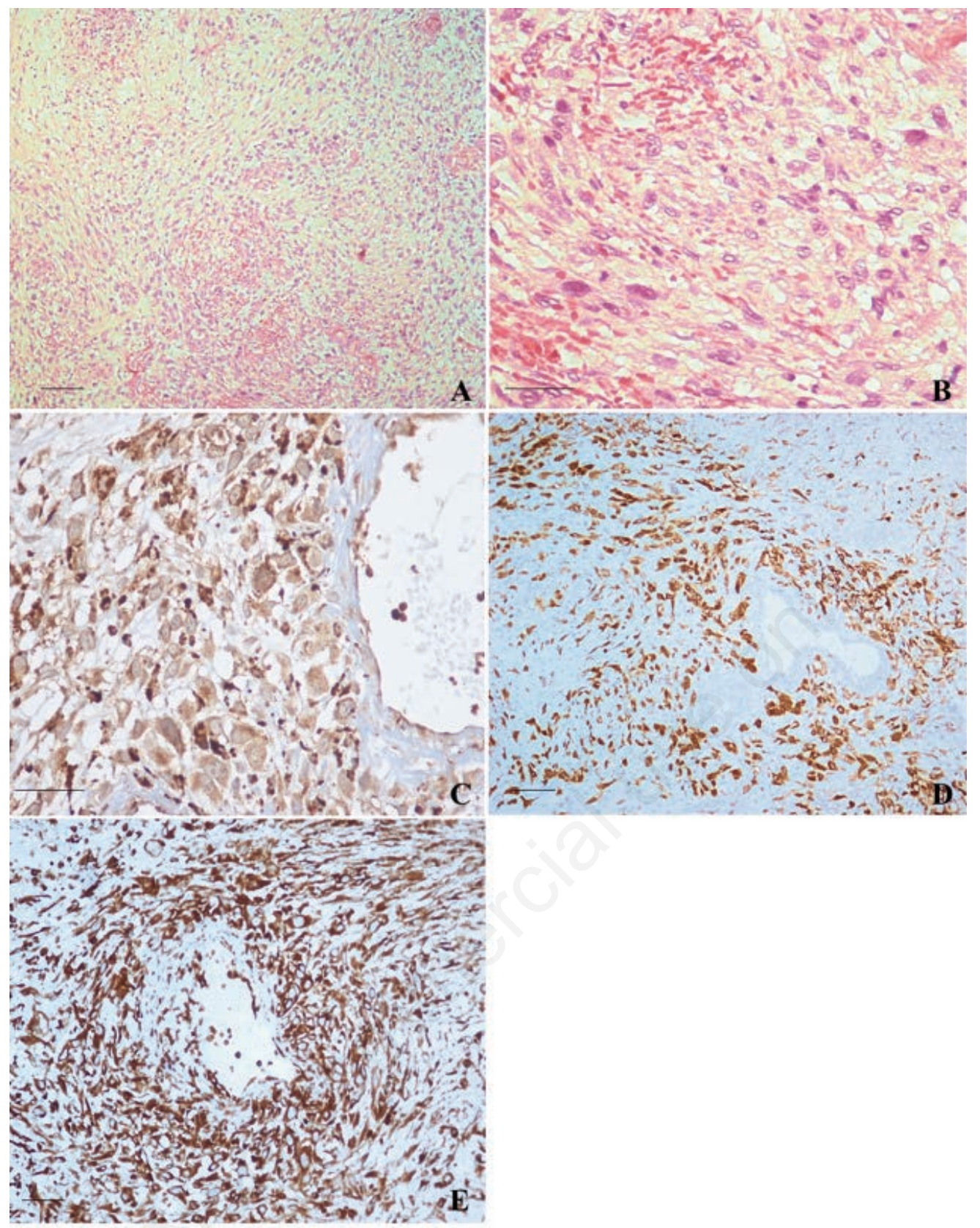

Figure 2. Histological section of the verrucous white plate stained by hematoxin and eosin showed features associated to the hyperplasia of atypical epithelial cells $(\mathrm{A}, 100 \mathrm{x})$ dispersed in the dermis $(\mathrm{B}, 400 \mathrm{x})$. Histological skin sections were positive for the markers $\mathrm{CD} 68$ $(C, 400 x)$, cytokeratin $(D, 100 x)$ and vimentin $(E, 200 x)$.

\section{References}

1. Brito AC de, Bittencourt M de JS. Chromoblastomycosis: an etiological, epidemiological, clinical, diagnostic, and treatment update. An Bras Dermatol 2018;93:495-506.

2. Rojas OC, González GM, MorenoTreviño M, Salas-Alanis J. Chromoblastomycosis by Cladophialophora carrionii Associated with Squamous Cell Carcinoma and
Review of Published Reports. Mycopathologia 2015;179:153-7.

3. Belda W, Criado PR, Domingues Passero LF. Case Report: Treatment of Chromoblastomycosis with Combinations including Acitretin: A Report of Two Cases. Am J Trop Med Hyg 2020. Available from: http:/www.ncbi.nlm.nih.gov/pubmed/3 2815507

4. Bao F, Wang Q, Yu C, et al. Case Report: Successful Treatment of
Chromoblastomycosis Caused by Fonsecaea monophora in a Patient with Psoriasis Using Itraconazole and Acitretin. Am J Trop Med Hyg 2018;99:124-6.

5. Urs A, Kumar P, Uniyal A, et al. Sarcomatoid carcinoma: A clinicopathological profile of two cases with diagnostic emphasis. Contemp Clin Dent 2018;9:164.

6. Folpe AL, Cooper K. Best practices in diagnostic immunohistochemistry: 
pleomorphic cutaneous spindle cell tumors. Arch Pathol Lab Med 2007;131:1517-24.

7. Maeda D, Fujii A, Yamaguchi K, et al. Sarcomatoid Carcinoma with a Predominant Basaloid Squamous Carcinoma Component: The First Report of an Unusual Biphasic Tumor of the Ureter. Jpn J Clin Oncol 2007;37:878-83.

8. Azevedo CMP, Marques SG, Santos
DWC, et al. Squamous cell carcinoma derived from chronic chromoblastomycosis in Brazil. Clin Infect Dis 2015,60:1500-4

9. Gon A dos S, Minelli L. Melanoma in a long-standing lesion of chromoblastomycosis. Int J Dermatol 2006;45:13313.

10. Caplan RM. Epidermoid carcinoma arising in extensive chromoblastomycosis. Arch Dermatol 1968;97:38-41.
11. Verma S, Thakur BK, Raphael V, Thappa DM. Epidemiology of Subcutaneous Mycoses in Northeast India: A Retrospective Study. Indian J Dermatol 2020;63:496-501.

12. Bonifaz A, Carrasco-Gerard E, Saúl A Chromoblastomycosis: clinical and mycologic experience of 51 cases. Mycoses 2001;44:1-7. 